\title{
Differential Speed Steering Control for Four-Wheel Independent Driving Electric Vehicle
}

\author{
Xiaodong Wu, Member, IACSIT, Min Xu, and Lei Wang
}

\begin{abstract}
The increasing prospect of electric drive vehicles has led many different control schemes to apply on the driving system. In this paper, differential speed steering which do not use traditional steering mechanism is studied on a four-wheel-driving vehicle. By comparison with the traditional Ackerman-steered vehicle, differential-steered method showed its advantage on the simple structure. Since the steering performance is just related with the velocities of four wheels, the strategy of differential speed on each motor is the major challenge. With the analysis of kinematic model of the vehicle, the relation between the turning behavior and the wheel parameters are investigated. A steering speed control method is proposed to get a steady turning performance during the acceleration or deceleration. The control strategy is implemented in a simulation to verify its rationality.
\end{abstract}

Index Terms-Differential speed steering, four wheel independent driving, electric vehicle.

\section{INTRODUCTION}

With the rapid development of electric vehicles (EVs), it has been commonly recognized that EVs are inherently more suitable to realize novel control over conventional internal combustion engine vehicles (ICEVs). Especially for the EVs with in-wheel motors installed in each tire can realize distributed driving system. The use of separate traction motors at each wheel implies that torque to each drive wheel can be controlled independently. By making full use of the excellent control capabilities of electric drive systems, EVs could not only be clean, but also be able to achieve better maneuverability that cannot be reached by conventional ICEVs.

In traditional ICEVs, a steering mechanism is indispensable for the turn motion. However, by using the merit of individual wheel traction motors on the electric drive vehicles, it opens up the possibility of using a differential speed steering systems [1]. A simple scheme is shown in Fig. 1. Since gearbox, retarder, transmission and steering mechanism are canceled from the four in-wheel motors independent drive vehicle, it has flexible layout and more efficient driving system. Using four in-wheel motors independently to drive vehicle, the steering of the vehicle is achieved by the differential speed controls on four wheels. The minimum turning radius of differential speed steering can

Manuscript received October 15, 2012; revised December 23, 2012. This work was supported by the National Post-doctoral Science Founda-tion of China under Grant 108804

$\mathrm{X}$. Wu, M. Xu, and X. Liu are with Institute of Automotive Engineering, School of Mechanical Engineering, Shanghai Jiaotong University, 800, Rd. Dongchuan, China (e-mail: xiaodongwu@sjtu.edu.cn, mxu@sjtu.edu.cn, wanglei19880307@126.com). be zero when a spot turn is performed, which is impossible by traditional steering method.

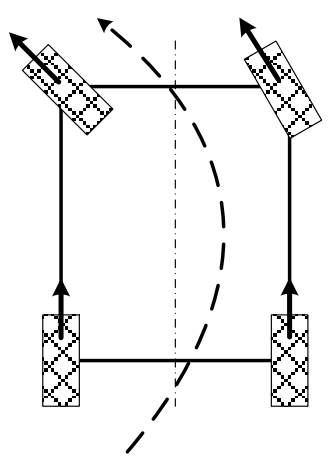

(a) Ackerman-steered vehicle

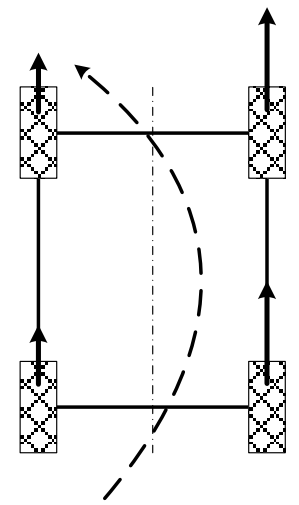

(b) Differential-steered vehicle
Fig. 1. Two kinds of steering methods

Differential speed steering is widely used in the navigation of mobile robot [2]-[4]. The steady steering trajectory of the mobile robot during the steering is not a main consideration, while the human-driving vehicle should take into account. Due to identical steering mechanisms, wheeled and tracked skid-steered vehicles share many properties [5], [6]. Many of the difficulties associated with modeling and operating in these kinds of skid-steered vehicles arise from the complex interaction between wheel and terrain. For Ackerman-steered vehicles, the wheel motions may often be accurately modeled by pure rolling, while for differential-steered vehicles, in general, are modeled by curvilinear motion, the wheels roll and slide at the same time [7]. This makes it difficult to develop kinematic and dynamic models, which accurately describe the motion characteristic. In [8] the comparison of differential-steering and Ackerman-steering for mobile robot is discussed. Vehicle can be steered through differential braking which is to get a speed difference in similar thought [9].

To obtain the desired turning radius is a difficult issue during the differential speed steering. Since the steering mechanism is using to implement the turn motion of traditional vehicle, the change of the wheel speed has no influence on the performance of turn motion. However, the turn motion during the differential speed steering is strongly coupled with the wheel speeds. In this paper, the objective is to analyze the behavior of differential speed steering applied to a fourwheel-driving electric vehicle when the inner and the outer wheel are given different speeds. To investigate the steering performance and behavior, the control model of the differential speed steering is studied. Due to complex tire/ground interactions and kinematic constraints, high speed driving will not be considered in this paper. A kinematic model based on 
the geometrical relationship for vehicle is figured out. Based on the vehicle model, Steering Speed Control (SSC) is proposed to keep the turning curve in desired value during acceleration and deceleration driving. The variable quantity of left wheels and right wheels are concluded that should satisfy a proportional relation. Differential speed steering control strategy for four in-wheel motors independent drive vehicle is performed to verify rationality of this method by simulation.

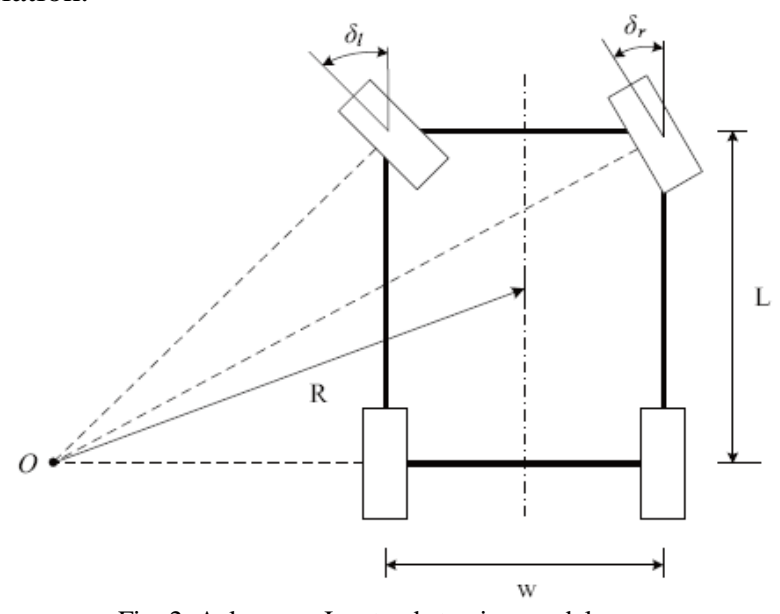

Fig. 2. Ackerman-Jeantand steering model.

This paper is organized as follows. Traditional steering method and the proposed differential speed steering is compared in Section II. The dynamic model and kinematic relationship of the differential speed steering is introduced in Section III. In the Section IV, to get a stable performance of steering, the control strategy of the proposed model is discussed. The conclusion and some discussions of future work are described in Section V.

\section{DiFFERENTIAL SPEED STEERING}

\section{A. Traditional Steering Method}

According to the Ackerman-Jeantand model [10] of steering when vehicle is running in a low speed, as be shown in Fig. 2, we define $L$ is the distance between front and rear wheel, $W$ is the track width of the vehicle, $\delta$ is average steering angle of two front wheels.

Depending on the above analysis, when the vehicle is steered with an angle $\delta$, the turning radius $R$ can be expressed by:

$$
R=\frac{L}{\tan \delta}
$$

From the Ackermann-Jeantand model, the kinematic geometrical relation of the vehicle parameters during the steering are expressed. The speed distributive relationship is achieved by balancing the force of the four wheels. It can be found that the turning radius of the vehicle is mainly influenced by the steering angle of the front wheels, and has no relationship with the advance speed of the vehicle.

\section{B. Differential Speed Steering}

Steering by differential speeds between the left and right wheels is widely used in the motion control of mobile robots. Benefited from the in-wheel-motor, four-wheel independent driving vehicle is suitable to take a similar control method. The motion direction of this kind of electric vehicle can be changed by rotating the left and right side wheels at different velocities, which is called differential speed steering. This steering mechanism makes the vehicle mechanically robust and simple which provides a new way to realize the motion control of electric vehicle.

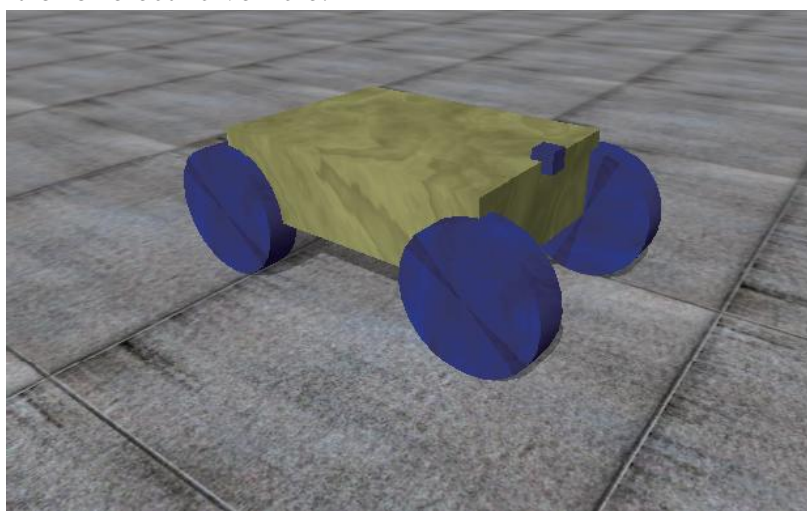

Fig. 3. ODE-based Simulation for the vehicle dynamic.

To analyze the differential speed steering control system, a simulator for four-wheel independent driving vehicle has been developed in an Open Dynamics Engine (ODE) environment. A screenshot is shown in Fig. 3. In the simulation, the four wheels are driving by independent motor and keeping the same orientation as the vehicle body. The vehicle is running on a firm ground surface, and four wheels are always in contact with the ground surface. The physical parameters of the simulated vehicle are given in Table I.

TABLE I: PhysicAl PARAMETERS OF THE SiMULATED VeHICLE

\begin{tabular}{cc}
\hline \hline Body Length $L:$ & $2.6 \mathrm{~m}$ \\
\hline Body Width $\mathrm{W}:$ & $1.4 \mathrm{~m}$ \\
\hline Body Hight $\mathrm{H}:$ & $1.3 \mathrm{~m}$ \\
\hline Radius of wheel: & $0.33 \mathrm{~m}$ \\
\hline Weight of body $\mathrm{m}_{b}:$ & $400 \mathrm{~kg}$ \\
\hline Weight of wheel $\mathrm{m}_{w}:$ & $25 \mathrm{~kg}$ \\
\hline Total Weight $\mathrm{m:}$ & $500 \mathrm{~kg}$ \\
\hline Friction coefficients: & 0.8 \\
\hline \hline
\end{tabular}

To simplify, two wheels on each side of vehicle is assumed to rotate at the same speed which is similar to the tracked vehicle. For the differential speed steering, the turning direction is determined by the side which has higher speed. If we define $\Delta \omega=\omega_{R}-\omega_{L}$, the vehicle will turn left when $\Delta \omega>0$, and the vehicle will turn right when $\Delta \omega<0$. To get desired speed difference, the control strategy can be implemented by three methods. One is increasing the speed of outer wheel, another one is decreasing the inner wheel speed, and the third one is speeding up the outer wheels and slowing down inner wheels simultaneously. Generally, from safety perspective, the vehicle speed is required to be slow down or be steady during the steering motion. So the latter two methods by decreasing the inner wheel speed will be considered in most situations. Three turning trajectories under different wheel speed are shown in Fig. 4. It can be found the turning radius is 
increased corresponding to the decrease of the speed difference $\Delta \omega$.

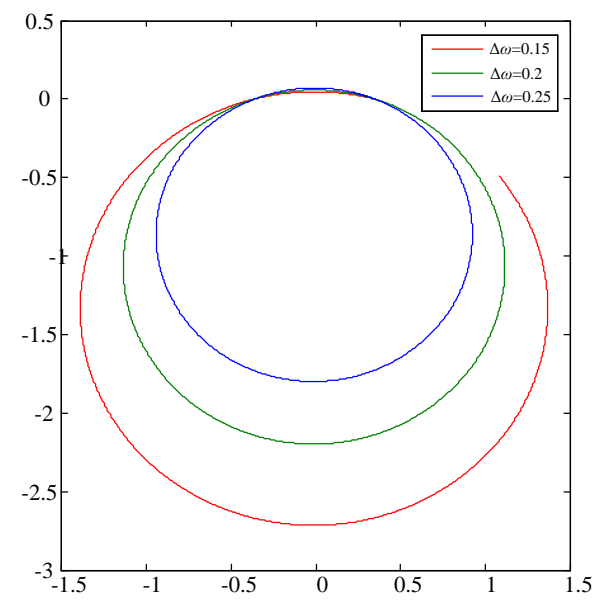

Fig. 4. Trajectory of the four-wheel-driving vehicle under different speed between left and right wheels $\left(\omega_{R}=0.3\right)$.

\section{MOdeLING OF VeHICLE STEERING}

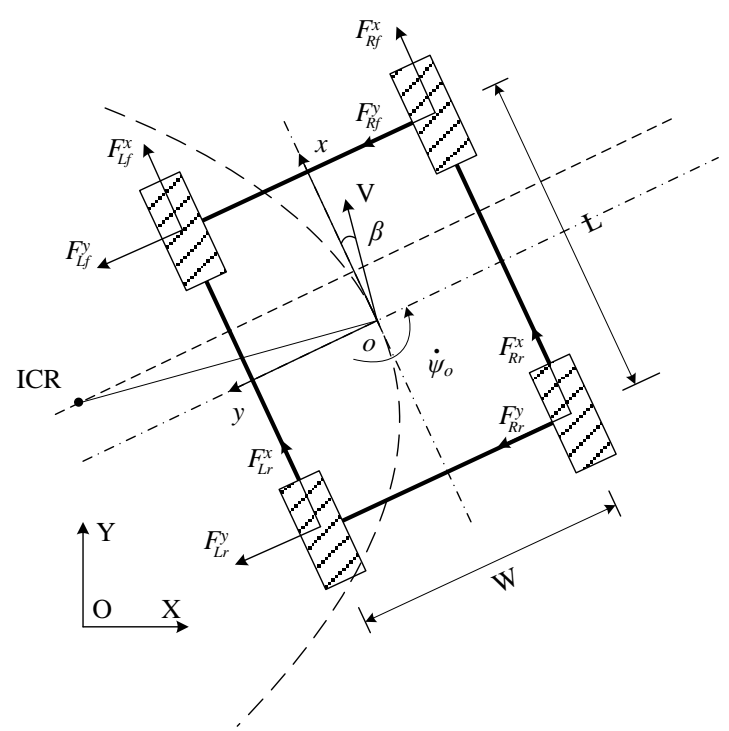

Fig. 5. Schematic of the kinematics model for a skid-steered vehicle.

A kinematic model of a differential-steered vehicle which maps the wheel velocities to the vehicle velocities is very important during the development of control strategy. To mathematically analyze the dynamic models of the differential speed steering, a schematic of four-wheel-driving vehicle moving at constant velocity about an ICR (instantaneous centers of rotation) is shown in Fig. 5. The local coordinate frame, which is attached to the body center of gravity, is denoted by $L(\mathrm{x}, \mathrm{y}, \mathrm{z})$, where $x$ is the lateral coordinate, and $y$ is the longitudinal coordinate.

\section{A. Vehicle Dynamic}

The mass center of the vehicle is assumed at the geometric center of the body frame. The 3-DOF dynamic equations in the body frame $L(\mathrm{x}, \mathrm{y}, \mathrm{z})$ can be written in equation (2), where the $F^{\mathrm{x}}$ and $F^{\mathrm{y}}$, indicate the longitudinal force and the lateral force. In the suffixes to each force $F$, the first letter refers to the left or right and the second to the front or rear. For example, $F_{L f}$ refers to the force acting on the left front wheel. $m$ and $I$ are the mass and moment of inertia of the vehicle. $W$ and $L$ are the width and length of the vehicle.

$$
\begin{aligned}
& m \ddot{x}=F_{L f}^{x}+F_{R f}^{x}+F_{L r}^{x}+F_{R x}^{x}+m \dot{\psi}_{o} \dot{y} \\
& m \ddot{y}=F_{L f}^{y}+F_{R f}^{y}+F_{L r}^{y}+F_{R x}^{y}+m \dot{\psi}_{o} \dot{x} \\
& I \ddot{\psi}_{o}=\left(F_{L f}^{x}+F_{R f}^{x}+F_{L r}^{x}+F_{R x}^{x}\right) \frac{W}{2} \\
& +\left(F_{L f}^{y}+F_{R f}^{y}+F_{L r}^{y}+F_{R x}^{y}\right) \frac{L}{2}
\end{aligned}
$$

In Fig. 5, signs such as $\beta$ denote the angle between the body axis and the moving direction of the vehicle. It is also can be taking as the sideslip angle of the tire which refers to the angle formed by the traveling direction and the surface of revolution of the tire. If the wheel radius is defined as $r$, the longitudinal and lateral force to the tire expressed in equation (2) is expanded in the following equations:

$$
\begin{aligned}
& J \dot{\omega}=T-F^{x} r \\
& F^{y}=C_{\alpha} \beta
\end{aligned}
$$

where $T$ is the driving/braking torque of the motor, $J_{\omega}$ is wheel inertia, $\omega$ is wheel angular velocity, $C_{\alpha}$ is the cornering power of the individual tires.

\section{B. Steering Kinematic}

ICR denote the instantaneous center of rotation of the vehicle body in Fig. 5. Based on the previous study, it is believed the ICR is laid on a line parallel to the y-axis [2], [7], [8]. In the local body coordinate, the longitudinal location $I C R_{x}$ and the lateral location $I C R_{y}$ satisfies the following constraints:

$$
\begin{gathered}
I C R_{x}=-\frac{v_{o y}}{\dot{\psi}_{o}} \\
I C R_{y}=\frac{v_{o x}}{\dot{\psi}_{o}}
\end{gathered}
$$

where $v_{o x}$ and $v_{o y}$ are the velocity of the mass center of vehicle body in longitudinal direction and lateral direction.

Define $v_{R}$ and $v_{L}$ be center linear velocities of right and left vehicle wheels shown in Fig. 6 . The radius of the turn can be calculated from similar triangles:

$$
\begin{aligned}
& v_{o x}=\frac{v_{L}+v_{R}}{2} \\
& \dot{\psi}_{o}=\frac{v_{L}-v_{R}}{W} \\
& I C R_{y}=\frac{W}{2} \frac{v_{L}+v_{R}}{v_{L}-v_{R}}
\end{aligned}
$$

The instantaneous turning radius of the vehicle can be expressed by

$$
R_{o}=\sqrt{I C R_{x}^{2}+I C R_{y}^{2}}
$$

In the local body coordinate, using equation (4) and 
equation (5), the kinematic relationship between the whole vehicle and the wheel speed can be summarized as:

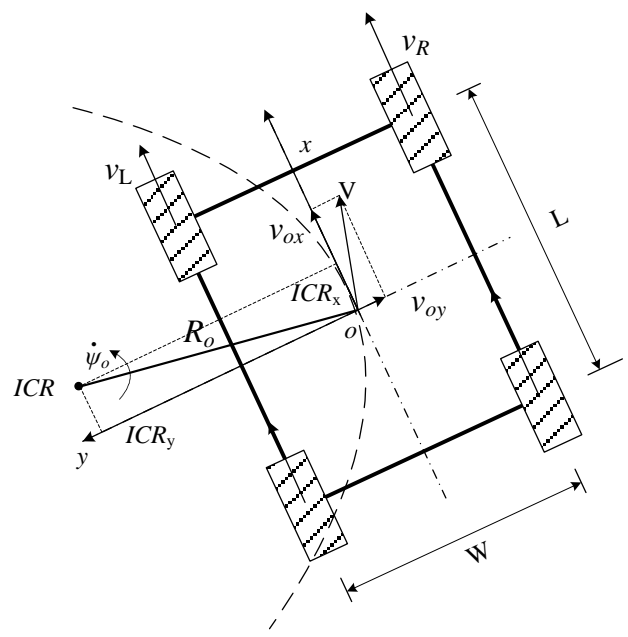

Fig. 6. Simplified kinematic model of the differential speed steering.

$$
\left(\begin{array}{c}
v_{o x} \\
v_{o y} \\
\psi_{o}
\end{array}\right)=\left(\begin{array}{cc}
\frac{1}{2} & \frac{1}{2} \\
\frac{I C R_{x}}{W} & -\frac{I C R_{x}}{W} \\
\frac{1}{W} & -\frac{1}{W}
\end{array}\right)\left(\begin{array}{l}
v_{L} \\
v_{R}
\end{array}\right)
$$

Wheel slip plays a critical role in the behavior of the differential speed steering. When the vehicle turns, the longitudinal slip of the wheels on both sides will be different. For the outer wheel, the wheel rotate speed is greater than the wheel line speed due to acceleration, and for the inner wheel, the wheel rotate speed is smaller than the wheel line speed due to deceleration. The longitudinal slip will be greater than zero in the inner wheel while be less than zero in the outer wheel. To obtain the desired turning radius and the yaw rate, the difference between the outer and inner speed must be controlled in real time.

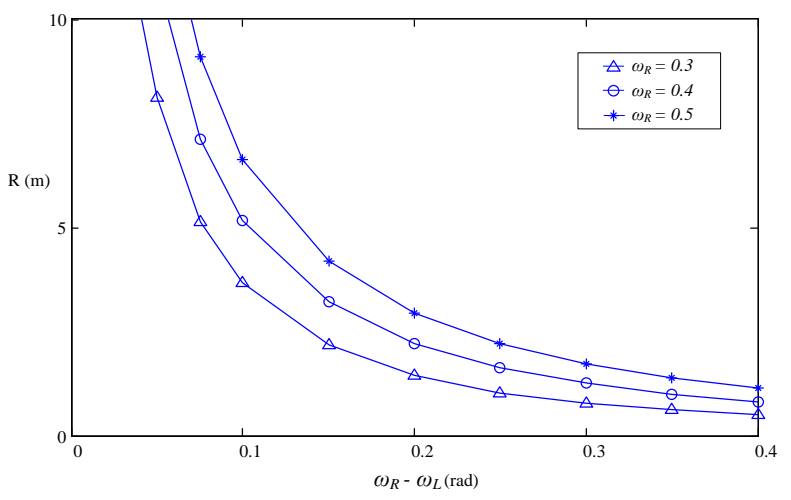

Fig. 7. Relation between the turning radius and wheel speed.

\section{CONTROL FOR DifFERENTIAL SPEED STEERING}

From the analysis of the kinematic relation during the differential speed steering, the turning radius has nonlinear relation with the speed difference between the wheels on both sides. Here a group of turning experiment under different initial speed is carried out. The results are shown in Fig. 7. It can be found that desired turning radius is difficult to calculate from this complex nonlinear relationship.

To figure out the relation between the turning radius and the rotation speed of wheels, we introduce a nondimensional variable $\gamma$ as the ratio of sum and difference of left-side and right-side wheel rotating velocities. It can be expressed as

$$
\gamma=\frac{\omega_{R}+\omega_{L}}{\omega_{R}-\omega_{L}}
$$

From the experimental results, we find that the turning radius has a linear relation with the parameter $\gamma$. A group of the experimental data shown in Fig. 8 has demonstrated such an observation. Here we set the right wheels rotating with a fixed speed, and the left wheel will change its speed from the zero to the same value as the right one. Therefore, we can use equation (12) to evaluate desired turning radius.

$$
R=K \gamma=K \frac{\omega_{R}+\omega_{L}}{\omega_{R}-\omega_{L}}
$$

where $K$ is a proportionality coefficient which can be calculated by a curve fit of the experimental data. If $\omega_{R}=\omega_{L}$, then $R=\infty$, and the vehicle is moving forward directly. If $\omega_{R}=$ $-\omega_{L}$, then $\mathrm{R}=0$, since the vehicle is performing in-place rotating about its center without any translational motion.

Since the turning radius during the differential speed steering is strongly coupled with the wheel speeds on both sides, the steering performance is sensitive to the real-time velocity of vehicle. During the steering, the acceleration or deceleration of vehicle with simple speed variation will make an oversteering or understeering. To keep stable turning performance, the change of the speed in the both right-side and left-side wheels should satisfy a proportional relationship. If we define the change of the wheel speed on both side as $\Delta x$ and $\Delta y$, to keep the turning radius during differential speed steering, the following equation should be satisfied:

$$
\frac{\omega_{R}+\Delta x+\omega_{L}+\Delta y}{\omega_{R}+\Delta x-\omega_{L}-\Delta y}=\frac{\omega_{R}+\omega_{L}}{\omega_{R}-\omega_{L}}
$$

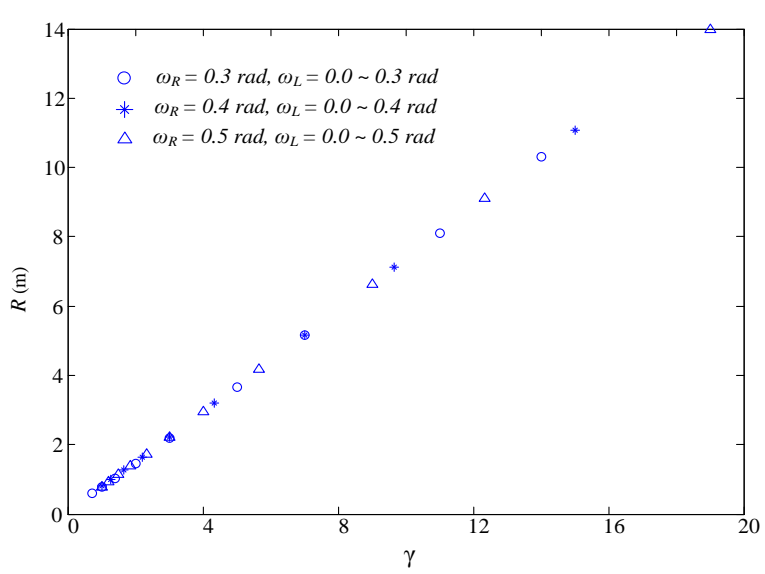

Fig. 8. Relation between the turning radius and wheel speed parameter $\gamma$.

After simplification, if the change of the wheel speed $\Delta x$ and $\Delta y$ satisfy the proportional relationship in equation (14), the turning radius can keep the same. To facilitate the following discussion, we call this Steering Speed Control (SSC).

$$
\frac{\Delta x}{\Delta y}=\frac{\omega_{R}}{\omega_{L}}
$$


In Fig. 9, we show the steering trajectories of simulated vehicle with SSC and without SSC. From the black circle, the speed of vehicle is changed for acceleration or deceleration until it reach white circle. An identical variable quantity $(\Delta x=$ $\Delta y=0.3 \mathrm{rad}$ ) has been added on each wheel for the acceleration trajectory. Meanwhile, for the braking trajectory each wheel has an identical decrease of the speed $(\Delta x=\Delta y=$ $-0.3 \mathrm{rad})$. From the differential speed steering without SSC, it can be found that the vehicle is oversteering or understeering during the acceleration and deceleration. While the change of the wheel velocity is based on the ratio relation in equation (14), the vehicle can moving in the desired steering circle steadily.

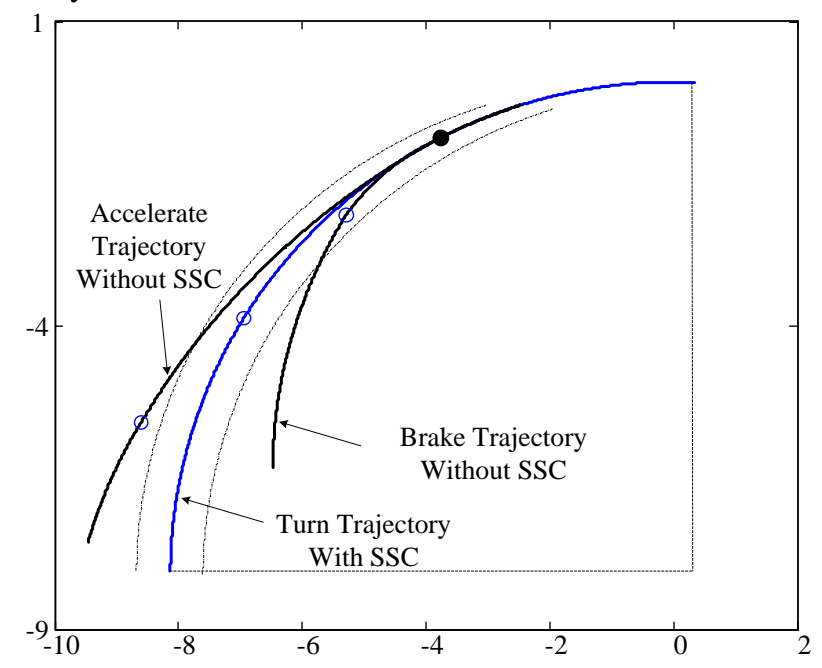

Fig. 9. Steering trajectories of the vehicle with SSC and without SSC.

\section{CONCLUSIONS AND FUTURE WORKS}

\section{A. Conclusions}

By utilizing the benefits of the independent motor driving electric vehicle, differential speed steering is studied to replace traditional mechanical steering in this paper. When the inner and the outer wheel are given different speeds, the steering behavior of the four-wheel-driving electric vehicle is analyzed in an ODE-based simulation platform. To solve the problem of changed turning radius during the velocity alteration, the kinematic model of steering based on the geometrical relationship for vehicle is figured out. Based on the vehicle model, Steering Speed Control (SSC) is proposed to keep the turning curve in desired value during acceleration and deceleration driving. The simulated results also verify the rationality of control strategy for differential speed steering in four in-wheel motors independent drive vehicle.

\section{B. Future Works}

During the differential speed steering with four wheels, the minimum turning radius can be zero for a pivot steering. But greater power and torque are required as a greater sideslip is encountered. Wheel slip plays a critical role in kinematic and dynamic modeling of the steering. Understanding the effect slip of the differential-steering, we can optimize the torque control of the motor to decrease the power consumption and tyre wear. In the future work, the wheel/ground interactions which directly provide traction and braking forces that affect the steering stability and turning radius will be studied.

\section{REFERENCES}

[1] L. Zhai and S. Dong. "Electronic Differential Speed Steering Control for Four In-wheel Motors Independent Drive Vehicle," in Proc. the 8th World Congress on Intelligent Control and Automation, Taipei, China, pp. 780-783, 2011

[2] J. Yi, H. Wang, J. Zhang, D. Song, S. Jayasuriya and J. Liu. "Kinematic Modeling and Analysis of Skid-Steered Mobile Robots With Applications to Low-Cost Inertial-Measurement-Unit-Based Motion Estimation," IEEE Transactions on Robotics, vol. 25, no. 5, pp. 1087-1097, 2009

[3] W. Yu, O. Chuy, E. G. Collins, and P. Hollis, "Analysis and Experimental Verification for Dynamic Modeling of A Skid-Steered Wheeled Vehicle," IEEE Transactions on Robotics, vol. 26, no. 2, pp. 340-353, 2010

[4] K. Kozlowski and D. Pazderski, "Modeling and control of a 4-wheel skid-steering mobile robot," Int. J. Appl. Math. Comput. Sci., vol. 14, no. 4, pp. 477-496, 2004

[5] J. Y. Wong, Theory of Ground Vehicles, 3rd ed. New York: Wiley, 2001.

[6] K. R. Weiss, "Skid-steering," Auto. Eng., pp. 22-25, 1971.

[7] J. Y. Wong and C. F. Chiang, "A general theory for skid steering of tracked vehicles on firm ground," Proceedings of the Institution of Mechanical Engineers, Part D: Journal of Automobile Engineering, vol. 215 , no. 3, pp. 343-355, 2001

[8] B. Shamah, "Experimental Comparison of Skid Steering Vs. Explicit Steering for a Wheeled Mobile Robot," Master Thesis, The Robotics Institute, Carnegie Mellon University, Pittsburgh Pennsylvania, USA, 1999.

[9] T. Pilutti, G. Ulsoy, and D. Hrovat. "Vehicle Steering Intervention Through Differential Braking," in Proc. American Control Conference, Washington, USA, pp. 1667-1671, 1995.

[10] J. Ackermann and W. Sienel, "Robust yaw damping of cars with front and rear wheel steering." IEEE Transactions on Control Systems Technology, vol. 1, no. 1, pp. 15-20, 1993.

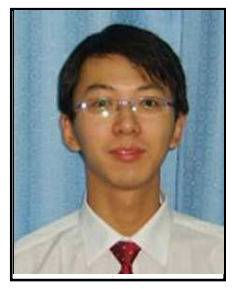

Xiaodong Wu received the B.Eng. and M.Eng. degree in Mechanical \& Electronic Engineering Science from China University of Petroleum, Beijing in 2008, and the Ph.D. degree from Ritsumeikan University, Japan in 2010, for research on the bio-inspired control system of the snake-like robots. He is currently working as a Postdoctoral Researcher in the Department of Mechanical Engineering at Shanghai Jiao Tong University. His research interests include dynamic control of electric vehicles, bio-inspired robots, and automotive electronics. He is a member of IEEE, IACSIT.

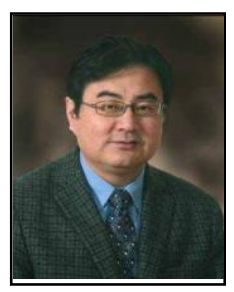

Min Xu received the Bachelor's degree of the Marine Powertrain Engineering, from Shanghai Jiao Tong University in 1983, and M.Eng. and Dr.Eng. degree in Transport Phenomena Engineering from University of Hiroshima in 1987 and 1991. He worked as postdoctoral researcher from 1991 to 1992 , and as a research staff from 1992 to 1995 in Carnegie Mellon University. Since 1995 he worked in GM, Delphi, Ford, Visteon respectively until 2003. From 2003 to 2006, he joined to Chery Automobile Company and worked as Vice President. He is currently working as Assistant President, Director of Institute of Automotive Engineering in Shanghai Jiao Tong University. His research interests include spray and combustion, combustion system for internal combustion engines, design and development for vehicle engines and vehicle integration and development. He is Deputy Director of National Engineering Laboratory for Automotive Electronic Control Technology. He also is an expert committee for "863" Mega Project of Energy-efficient and New Energy Vehicle. He is a SAE Fellow, and serves many societies and conferences as committee members and chairs.

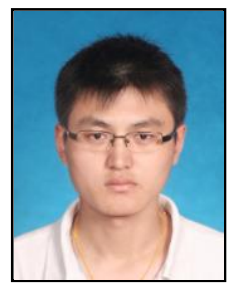

Wang Lei received the Bachelor's degree in the Department of Dynamic \& Mechanical Engineering from Wuhan University in 2011. He is currenty pursueing M.S. degree in the Department of Mechanical Engineering at Shanghai Jiao Tong University. His research interests include embedded control system development, and vehicle dynamic research. 